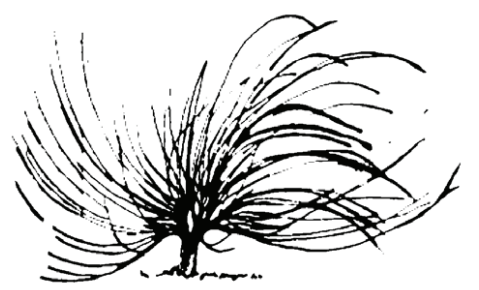

\title{
La relación existente entre la metodología utilizada por un profesor de ciencias y la motivación que presentan los estudiantes de un grupo de noveno año
}

\author{
Andrea Alvarado Arguedas ${ }^{1}$ \\ Universidad Nacional de Costa Rica \\ Heredia, Costa Rica \\ mandrea_0069@hotmail.com \\ Ricardo Salas Sánchez ${ }^{2}$ \\ Universidad Nacional de Costa Rica \\ Heredia, Costa Rica \\ rsalas89@gmail.com
}

\begin{abstract}
Resumen
En el ámbito educativo, tradicionalmente la ciencia se ha concebido una materia totalmente expositiva dentro del $\mathrm{Cu}$ rriculum de las instituciones, generando problemas graves en el proceso de enseñanza, puesto que, no se desarrolla el interés y motivación correcta que satisfaga las necesidades de los educandos produciendo problemas en el desarrollo científico y tecnológico de nuestro país. Para constatar esta situación se realizó una investigación en una institución diurna privada del circuito 01 de la provincia de Heredia. Para la recopilación de la información se realizaron: tres observaciones al grupo en seis lecciones, una entrevista al docente, asimismo un cuestionario a cada estudiante del grupo para conocer su agrado y motivación por la metodologíade las clases de Ciencias, así como determinar cuáles
\end{abstract}

Recibido: 30 de noviembre de 2011 - Aprobado: 12 de marzo de 2012

1 Bachiller en Enseñanza de las Ciencias de la Universidad Nacional de Costa Rica.

2 Bachiller en Enseñanza de las Ciencias de la Universidad Nacional de Costa Rica. 
técnicas y recursos didácticos son las de más agrado para ellos. Por último se les aplicó a los estudiantes una escala de motivación con el fin de medir y determinar el interés del alumno en la materia y experiencias vividas dentro del proceso de aprendizaje.

Palabras clave: Metodología, motivación, aprendizaje, técnicas, recursos didácticos.

\begin{abstract}
:
In the education system, Science has always been considered a traditional lectures subject inside the curriculum institutions. This conception has provoked serious problems in the teaching process because students have not developed a proper interest and motivation that fulfill their learning needs provoking effects on the scientific and technological development of the country. In order to take note of the situation, a research was conducted at a private day institution in the 01 circuit of Heredia province. The information was gathered through different instruments: three six-lesson observations, a teacher interview, a students' questionnaire about their opinion on Science class methodology and the use of teaching resources. Finally, a motivational scale was given to students to measure and determine their interest on the subject and their lived experiences as part of their learning process.
\end{abstract}

Keywords: Metodología, motivación, aprendizaje, técnicas, recursos didácticos.

\title{
Introducción
}

Oon el fin de estudiar la relación existente entre la metodología utilizada por un profesor de ciencias y la motivación que presentan los estudiantes, se realizó una investigación en una institución diurna privada del circuito 01 de la provincia de Heredia, en la cual se tomó como muestra un grupo de 25 estudiantes que cursan el noveno año, de igual forma al profesor que imparte la materia de Ciencias. Dichos estudiantes en su mayoría se caracterizan por provenir de zonas urbanas aledañas al colegio, cuya edad se encuentra entre los 14 
y 16 años y son de ambos sexos. Procedentes de hogares de condición económica media y alta.

Con esta investigación se pretendió conocer cómo influye la metodología que emplea el docente de Ciencias en la motivación de los estudiantes de un grupo de noveno año en un colegio privado; para ello se analizó la vinculación que presenta la metodología empleada por el docente de Ciencias y su influencia en la motivación de los estudiantes de noveno año durante las clases de dicha materia.

Concretamente se describió la metodología empleada por el docente de Ciencias en sus lecciones, así como sus repercusiones en la motivación del grupo. De igual forma, se determinó cuáles son las estrategias metodológicas que incentivan a participar y a mantener la motivación de dichos estudiantes. Con el fin de establecer la relación existente entre las variables en estudio, se elaboró una propuesta didáctica para el tema La célula, que promueva la motivación en el proceso enseñanza aprendizaje en el nivel de noveno año.

La investigación nace a raíz de que la metodología empleada por algunos docentes no es la más adecuada para lograr un aprendizaje significativo, asimismo, mantener la motivación de los educandos en la asignatura de Ciencias. Por ello es de suma importancia conocer cuáles son las metodologías más eficaces que influyen en el aprendizaje y la motivación de los educandos.

El docente debe conocer la manera más idónea para el aprendizaje del grupo en general y de cada estudiante, para que el conocimiento suceda de una forma bidireccional, participativa, activa e interesante. Para ello es necesario que el docente se mantenga en constante actualización y renovación de sus técnicas y conocimientos, ya que sus clases se pueden volver rutinarias y monótonas para sus estudiantes, influyendo positiva o negativamente en la motivación del estudiante en el salón de clases.

El tema de esta investigación es importante para conocer qué repercusiones tiene la metodología utilizada por el docente en la motivación hacia la materia en estudio por parte de los educandos, para que el aprendizaje de estos no sea visto como una obligación sino una experiencia agradable en su vida, donde pueda aprender y aplicar los conocimientos de forma interactiva a su realidad.

Este tema ha sido abordado anteriormente por diferentes autores, entre ellas se puede citar a Esquivel, Gutiérrez, Solís y Zúñiga (2005), quienes investigan cómo la motivación influye en la actitud y 
desempeño del estudiante, obteniendo cómo resultado que la motivación debe estar presente en ambos, ya que es una relación directa entre los actores de la educación, asimismo concluyen que el docente debe conocer cuáles son las metodologías que más incentivan a sus estudiantes a participar, y por ende obtener un aprendizaje significativo.

En el año 2008, Ávila, Cascante y Quesada nos indican que con mayor frecuencia los educadores desarrollan sus lecciones de forma tradicional y muchos de ellos no presentan interés por actualizarse con metodologías de mayor pertinencia para cambiar la acción en el aula y superar aspectos que se descuidan actualmente en la enseñanza de las ciencias. Además, se implementa un diseño de módulos de capacitación para optimizar los procesos de enseñanza y aprendizaje para los docentes de ciencias y biología utilizando los recursos físicos y naturales disponibles en una estación biológica.

De igual manera, Fernández y Orozco (2002) obtienen en su investigación que la metodología empleada por el docente tiene un papel importante en la motivación de los estudiantes por la materia, porque es mediante las técnicas y actividades creativas que el profesor logra involucrar al estudiante para mantener su atención. Además, deducen que la motivación en el aula se debe encontrar en todos los participantes del proceso, es decir, tanto el profesor como los estudiantes.

Con base en la literatura consultada, esta investigación buscó comprobar la relación que se da entre la motivación del estudiante y la metodología empleada por el profesor. Según Giroux (1990), la desmotivación que presentan los estudiantes es consecuencia de una "crisis educativa", la cual llega a nuestra aulas por medio del educador ya que éste se encuentra agobiado por el recargo de funciones,, así como la escasa cantidad de material didáctico, infraestructura y recursos económicos para poder impartir las lecciones a una gran cantidad de educandos.

\section{Referente teórico}

\section{El aprendizaje y la metodología didáctica de la enseñanza de las Cien- cias Naturales}

Cuando el ser humano realiza una o varias actividades con el objetivo de asimilar, conocer y observar un fenómeno de la naturaleza y adquirir un nuevo conocimiento, habilidad o capacidades lo que se 
denomina aprendizaje. En el ámbito pedagógico, la educación tiene como fin último lograr un aprendizaje en los estudiantes. Según el Diccionario de La Real Academia Española (2001), aprender significa: "Adquirir el conocimiento de algo por medio del estudio o de la experiencia".

Para Gagné (1997, p.19), "todo acto de aprendizaje requiere de la existencia de estados previamente aprendidos, donde se deben disponer de ciertas habilidades intelectuales y métodos de autoadministración que rijan la conducta por atender, almacenar y recuperar información así como organizar la solución del problema". Por ello, en el proceso de enseñanza se deben aprovechar los conocimientos previos y las experiencias de vida de los estudiantes, además el docente debe crear un ambiente favorable, una contextualización de contenidos y una adecuación de ellos acorde con la forma de aprendizaje de cada alumno. Todo ello con el fin de crear un aprendizaje significativo en el estudiante.

El modelo de aprendizaje significativo es propuesto por David Ausubel en 1960, que lo define como "un proceso por el que se relaciona una información con algún aspecto ya existente en la estructura cognitiva de un individuo y que sea relevante para el material que se intenta aprender" (Novak, 1968, p.71). El aprendizaje significativo permite que el estudiante vincule los contenidos con su realidad, desarrollando conocimientos que son útiles y aplicables en las diferentes situaciones de la vida.

Para lograr un aprendizaje significativo, el profesor debe utilizar una metodología idónea para el progreso cognitivo del estudiante. Actualmente, no hay una definición clara de metodología ya que no existe un proceso único y correcto para alcanzar los objetivos en la educación. Además, está limitada por la capacidad, el conocimiento y la creatividad del docente.

La metodología es el conjunto de momentos y técnicas lógicamente coordinados para dirigir el aprendizaje del alumno hacia determinados objetivos. Dentro de la pedagogía crítica la metodología, debe conducir a la construcción de conocimiento y el desarrollo de criticidad, que sean de utilidad en el contexto de los estudiantes, para que participen de la transformación del cambio social (Giroux, 1990).

De acuerdo con el Ministerio de Educación Pública (2005), en el programa de Biología se proponen diferentes metodologías, basadas en la clasificación de Nérice: 
Por la forma de razonamiento, los clasifica en deductivo (de lo general, universal a lo particular); inductivo (de la particular a lo general) y análogo (por establecimiento de relaciones).

Por las actividades de los alumnos, los clasifica en pasivo (cuando el más importante en el proceso es el profesor) y activo (cuando el estudiante desempeña todo su esfuerzo personal de creación o de búsqueda).

Por el trabajo de los alumnos, los clasifica en individual (se considera que cada alumno es un caso único e irrepetible); grupal (da énfasis a la socialización) y mixto busca explorar al máximo las posibilidades de cada educando, sin dejar de lado la socialización que capacita al estudiante para los trabajos en equipo. (p.73)

La metodología opera con una serie de técnicas y recursos didácticos, que facilitan el éxito del proceso de enseñanza-aprendizaje. Para Esquivel et al. (2005) "una técnica didáctica es la forma especial que el maestro tiene de seleccionar y dirigir actividades docentes dentro de su clase" (p.29), por ejemplo: discusión en grupos, mesa redonda, expositiva, entre otros. Por su parte, los recursos didácticos son todos aquellos medios empleados por el docente en el proceso educativo que guía para apoyar, acompañar, complementar o evaluar los contenidos (Grisolía, s.f.), como la pizarra, carteles, equipo multimedia, entre otros.

\section{La motivación en la enseñanza de las Ciencias Naturales}

Se considera la motivación como un impulso que parte del organismo, como una atracción que emana del objeto (metodología) y actúa sobre el individuo (estudiante), además está compuesta de necesidades, deseos y expectativas y constituye un paso previo al aprendizaje ya que es el motor del mismo, de ahí que se puede afirmar que no hay aprendizaje sin motivación (Prot, 2005).

Al estudiar la motivación, debe diferenciarse el motivo (estímulo interno), del incentivo (estímulo externo), por esto la motivación se divide en dos tipos intrínseca y extrínseca.

La motivación intrínseca es claramente motivación, la persona la realiza por su propio placer y satisfacción, mientras que la motivación extrínseca es externa, no existe ya que se da por incentivación, aparece 
cuando lo que atrae no es la acción que se realiza en sí, sino lo que se recibe a cambio, un premio (Gilbert, 2005).

Por eso muchos autores afirman que el profesor no motiva, solamente incentiva, puesto que el estudiante ejecuta la actividad o acción propuesta a cambio de un puntaje. No obstante, tanto los hechos o motivos que el docente realice, así como los incentivos que utiliza, van de la mano y no puede existir uno sin el otro y deben estar presentes durante todo el proceso de aprendizaje.

Es importante mencionar que para que el proceso de enseñanza aprendizaje cumpla con los objetivos propuestos es necesario que siempre esté presente la motivación en el ambiente del aula.

El docente es la persona que se dedica profesionalmente a la enseñanza. Además, tiene la función de la transmisión de valores, técnicas y conocimientos para facilitar el aprendizaje para que el alumno lo alcance de la mejor manera posible.

Como lo establece Díaz y Hernández (2002):

El papel del docente en el ámbito de la motivación se centrará en inducir motivos en sus alumnos en lo que respecta a sus aprendizajes y comportamientos para aplicarlos de manera voluntaria a los trabajos de clase, dando significado a las tareas escolares y proveyéndolas de un fin determinado de manera tal que los alumnos desarrollen un verdadero gusto por la actividad escolar y comprendan su utilidad personal y social. (p. 234)

Todo educador tiene como responsabilidad innovar constantemente su metodología que propicien el diálogo y reflexión entre los participantes del proceso con el fin de incentivar y promover un ambiente de aula y un proceso de aprendizaje ameno, en el cual el estudiante se sienta a gusto y motivado de lo que está aprendiendo, que el estudio no sea una obligación sino que este desee por sí mismo aprender cada vez más.

Según Esquivel et al. (2005), la gente influye en la gente ya sea de manera negativa o positiva, igualmente los educandos manifiestan un grado de dependencia hacia el profesor, es decir, si el profesor no se encuentra motivado en el momento de impartir las clases, jamás logrará motivar a sus estudiantes.

La relación entre estudiante y alumno es directa, el educador no puede ser un agente efectivo en el proceso de enseñanza aprendizaje sin la 
cooperación y participación de sus alumnos, es necesario que ambos estén comprometidos con el desarrollo de una clase activa y comunicativa.

En el año 2008, David Perkins expresó que el aprendizaje es posible cuando no se obliga al alumno y está presente una motivación para ello. Además, se le debe adecuar los métodos y contenidos, con una dificultad media, de acuerdo con sus capacidades. La dificultad está en saber qué es lo que se quiere enseñar y cómo presentar ese contenido al alumno de forma que le sea posible aprenderlo y que se sienta motivado para hacerlo. La motivación de los alumnos es una cuestión que en parte depende de ellos mismos, pero que en parte también puede depender del profesor y de cómo le presente el aprendizaje y le guíe a través de los procesos que lo forman.

\section{Métodos}

Este proyecto de investigación se sitúa dentro del paradigma naturalista y del enfoque cualitativo. Según Hernández, Fernández y Baptista (2006):

El enfoque cualitativo es aquél que utiliza la recolección de datos sin medición numérica para describir o afinar preguntas de investigación en el proceso de interpretación, además añaden que la recolección de los datos consiste en obtener las perspectivas y puntos de vista de los participantes (emociones, experiencias, significados y otros aspectos subjetivos), además resultan de interés las interacciones entre grupo, individuos y colectividades (p.5).

Asimismo, se basa en el tipo de investigación acción, la cual está relacionada con los problemas habituales que se dan en el contexto educativo, puede ser desarrollada por los mismos profesores o por alguien a quien ellos se lo encarguen (Elliot, 2000), quienes estudian los problemas concretos de las sociedades a fin de transformarlas, lo que obliga al investigador a pensar, reflexionar y actuar sobre la situación a fin de dar respuestas útiles a los problemas.

La población con la que se llevó a cabo la investigación fue un docente y veinticinco estudiantes, éstos últimos componen un grupo de noveno año de un colegio diurno del circuito 01 de la dirección regional de Heredia. El docente colaborador tiene a su cargo las lecciones de 
Ciencias del grupo en estudio, además tiene la función de ser el profesor guía de dichos estudiantes. Para este estudio, la muestra seleccionada fue elegida al azar.

Luego de tres observaciones consecutivas al grupo, se determinó el problema, por lo que se procedió a aplicar un cuestionario con preguntas abiertas y cerradas, además de un test de motivación dirigido a los estudiantes. También se realizó una entrevista no estructurada al profesor a cargo del grupo.

Los instrumentos fueron elaborados según las necesidades y objetivos de la investigación, los cuales se describen a continuación:

Tres observaciones directas en seis lecciones diferentes, con el fin de determinar un problema. Se trató de delimitarse a registrar los datos observados sin intervenir en la dinámica del aula, con el fin de evitar sesgos en la información requerida, además las expectaciones se registraban en un pequeño cuestionario que indicaba las principales pautas a observar.

Una vez discriminado el problema se realizó una entrevista al profesor, la entrevista realizada al profesor colaborador era semiestructurada puesto que, se formuló un diálogo guiado con una serie de preguntas entre el entrevistado y el entrevistador. En total se efectuaron ocho preguntas, con el objetivo de conocer cuál es la metodología que emplea en sus clases, si está consciente de las consecuencias en el uso de ésta y el porqué utiliza dicha metodología.

A los estudiantes se les aplicó un cuestionario, el cual tenía como visión conocer si a los estudiantes les agrada y motiva la metodología de las clases de Ciencias, así como determinar cuáles técnicas y recursos didácticos son los de más agrado para ellos.

Además, se les aplicó a los estudiantes una escala de motivación con el fin de medir y determinar el interés del alumno en la materia y experiencias vividas dentro del proceso de aprendizaje.

Por tanto, para el análisis de la información se utilizó como metodología central la adecuada triangulación de la información extraída a partir de los instrumentos aplicados a los diferentes sujetos de investigación, observaciones y teorías consultadas.

También para tener una mejor visión de los datos obtenidos se organizó la información por medio de matrices para las preguntas del cuestionario y del test que arrojaron datos significativos. Estas matrices al igual que otros datos relevantes fueron analizados para conseguir un 
mejor panorama sobre la relación existente entre la metodología empleada por el profesor y la motivación de los estudiantes.

\section{Resultados y análisis}

A continuación se presentan los resultados más significativos y representativos del trabajo de campo, el cual tiene como objetivo conocer la influencia de la metodología utilizada por el profesor en la motivación de los estudiantes.

Además, se presenta el análisis de los datos recolectados por medio de la aplicación respectiva de una entrevista al docente colaborador, un cuestionario y un test de motivación para todo el grupo.

\section{Matriz $\mathbf{N}^{0} 1$}

Conceptos más destacados de motivación según los estudiantes y el profesor de un colegio diurno privado del circuito 01 de Heredia

\begin{tabular}{|l|l|}
\hline \multicolumn{1}{|c|}{ Actores } & \multicolumn{1}{c|}{ Descripción } \\
\hline Estudiante 5 & Algo que nos da fuerza para seguir adelante. \\
\hline Estudiante 8 & Las ganas de hacer algo. \\
\hline Estudiante 9 & Algo que lo inspira a hacer cosas. \\
\hline Estudiante 18 & $\begin{array}{l}\text { Es incentivarnos a seguir adelante diciéndonos cosas positi- } \\
\text { vas e inspiradoras. }\end{array}$ \\
\hline Estudiante 23 & Algo que me da fuerzas para llegar a hacer algo. \\
\hline Profesor colaborador & $\begin{array}{l}\text { Es como un incentivo que se le puede dar a un estudiante para } \\
\text { que se supere y continúe adelante mostrándole sus capaci- } \\
\text { dades y virtudes. }\end{array}$ \\
\hline
\end{tabular}

Fuente: Cuestionario para estudiantes y entrevista al profesor colaborador.

Como se evidencia en los datos obtenidos, el concepto de motivación no está siempre claramente definido, puesto que los estudiantes lo definen como una emoción o sentimiento abstracto de impulso que los conduce a realizar un fin determinado, de igual forma el profesor lo cataloga como un sentimiento únicamente extrínseco y conductista, no obstante, Gilbert (2005) afirma que existen dos tipos de motivación (extrínseca e intrínseca), las cuales se encuentran relacionadas de forma directa. Por ello deben estar presentes en el proceso de enseñanza para obtener un aprendizaje significativo para que los estudiantes logren adquirir conocimientos que le sean útiles para su vida diaria, indiferentemente del campo de estudio en que se vaya a desempeñar. 


\section{Matriz $\mathbf{N}^{0} 2$}

Nivel de incidencia acerca del interés en los temas desarrollados en las clases de Ciencias

\begin{tabular}{|l|l|}
\hline \multicolumn{1}{|c|}{ Actores } & \multicolumn{1}{c|}{ Descripciones } \\
\hline $\begin{array}{l}\text { Estudiante1,2, 3, 5, 6, 7,8,10, } \\
12,13,15,18,19,20,23,25\end{array}$ & $\begin{array}{l}\text { Sí, porque me atraen la atención mucho/ porque nos } \\
\text { enseña cosas que necesitamos saber. }\end{array}$ \\
\hline Estudiante 4, 9,11,17,21,22 & $\begin{array}{l}\text { A veces/la mayoría de las veces/ algunos son intere- } \\
\text { santes otros aburridos. }\end{array}$ \\
\hline Estudiante 14, 16,24 & $\begin{array}{l}\text { No, porque es aburrido/ porque no tienen nada que } \\
\text { ver }\end{array}$ \\
\hline Profesor colaborador & $\begin{array}{l}\text { En mi opinión para la gran mayoría de los estudi- } \\
\text { antes es de mucho agrado y de mucho provecho. }\end{array}$ \\
\hline
\end{tabular}

Fuente: Cuestionario para estudiantes y entrevista al profesor colaborador.

Otra característica estimada es el interés de los alumnos hacia los temas de ciencias desarrollados ya que éste influye en la motivación de los estudiantes. La mayoría de ellos al igual que el profesor afirman que son importantes, puesto que se relaciona con la vida cotidiana y estos conocimientos pueden resultar necesarios en un futuro próximo, es decir, que son de provecho, sin embargo existen estudiantes con opiniones contrarias, por lo que puede considerarse una consecuencia del tipo de motivación que presentan dichos discentes, de manera que es importante la utilización de variadas actividades de enseñanza que despierten el interés y el deseo de aprender en cada uno de los estudiantes, pues cada persona aprende de manera diferente (Minnick \& Alvermann, 1994).

Matriz $\mathbf{N}^{0} 3$

Interés promovido hacia la asignatura de Ciencias de acuerdo a la metodología utilizada por el profesor

\begin{tabular}{|l|l|}
\hline \multicolumn{1}{|c|}{ Actores } & \multicolumn{1}{c|}{ Descripciones } \\
\hline Estudiante $1,5,6,7,9,11,16,23$ & No \\
\hline Estudiante $2,10,13,19,20,25$ & $\mathrm{Si}$ \\
\hline Estudiante $3,4,17,21,22,24$ & A veces/más o menos/tal vez \\
\hline Estudiante 8,12, & NR (no respondió) \\
\hline Estudiante 14 & $\begin{array}{l}\text { El profesor da muy bien la materia pero la verdad a } \\
\text { mí no me gusta }\end{array}$ \\
\hline Estudiante 15,18 & Un poco \\
\hline
\end{tabular}

Fuente: Cuestionario para estudiantes. 
En la matriz $\mathrm{N}^{\circ} 3$ se evidencia que la metodología utilizada por el profesor no es la más adecuada, puesto que no logra incentivar a la gran mayoría del grupo. De acuerdo con las observaciones realizadas, el profesor utilizó metodologías pasivas que no promueven la participación de los estudiantes, donde éste se convierte en un receptor de información sin permitir la construcción de su conocimiento. Según Lloyd (1970), el aprendizaje se realiza por razones de carácter personal, por eso el ser humano no aprende aquello que no le sea satisfactorio. Muchos de los alumnos no encuentran motivación en las Ciencias al no participar en el proceso de construcción de su conocimiento.

\section{Matriz $\mathbf{N}^{0} 4$}

Principales opiniones del profesor colaborador acerca de la influencia de la metodología en la motivación de los estudiantes

\begin{tabular}{|l|l|}
\hline \multicolumn{1}{|c|}{ Pregunta } & \multicolumn{1}{c|}{ Repuesta } \\
\hline 4 & $\begin{array}{l}\text { Claro motivar a un estudiante lo incentiva a superarse a mejorar, a } \\
\text { ser mejor persona }\end{array}$ \\
\hline 6 & $\begin{array}{l}\text { Sí, porque esto marca el grado de interés que puede tomar el estudi- } \\
\text { ante por la materia }\end{array}$ \\
\hline 7 & $\begin{array}{l}\text { - Los trabajos en grupo =incentivan el intercambio de ideas } \\
\text { - Antorchas= incentiva el espíritu de competencia } \\
\text { - Juegos= hacen las clases más divertidas }\end{array}$ \\
\hline 8 & $\begin{array}{l}\text { Es muy variado, esto debido a que algunos les gusta una cosa y a } \\
\text { otros otra. }\end{array}$ \\
\hline
\end{tabular}

Fuente: Entrevista al profesor colaborador

Según la matriz $\mathrm{N}^{\circ} 4$, el profesor está consciente de la importancia de la motivación en el proceso de enseñanza aprendizaje ya que cuando los estudiantes se encuentran motivados la clase genera un aprendizaje significativo en ellos. Sin embargo, en la observación realizada el profesor no utiliza una metodología activa y análoga, debido a la rutinaria planificación de sus clases. Otro aspecto que destaca el docente son los diferentes procesos por los que aprenden los estudiantes lo que conlleva a que la metodología no satisfaga las necesidades de aprendizaje de todos los estudiantes, esto concuerda con Martínez (s.f.): "Los alumnos no se motivan por igual, por lo que es importante buscar y realizar actividades motivadoras que impliquen mayor participación del alumno" (s.p). 
Podemos considerar que el interés hacia la ciencia se encuentra influenciado por la selección rigurosa de actividades (metodología) que respondan a las necesidades e intereses del educando. Por ello es de suma importancia conocer al estudiante para poder planear de la manera más apropiada.

\section{Matriz $\mathbf{N}^{0} 5$}

Técnicas y recursos didácticos empleados por el profesor

\begin{tabular}{|c|c|}
\hline Actores & Descripción \\
\hline Estudiantes & $\begin{array}{c}\text { Discusiones grupales, clases magistrales, ex- } \\
\text { posiciones, carteles }\end{array}$ \\
\hline Profesor & $\begin{array}{c}\text { Exposiciones, trabajos grupales, prácticas, } \\
\text { pequeñas investigaciones y libro de texto }\end{array}$ \\
\hline Observación no participante & Clases magistrales, fotocopias, libro de texto \\
\hline
\end{tabular}

Fuente: Guía de observación no participante, Cuestionario para estudiantes, Entrevista al profesor colaborador.

La matriz $\mathrm{N}^{\circ} 5$ nos expresa que el profesor imparte sus clases a través de las clases magistrales, pero de una forma conductista y errónea. Esta técnica permite que el docente transfiera sus conocimientos de una forma directa, llevando a los alumnos a reflexionar y descubrir las relaciones entre los diversos conceptos, formando una mentalidad crítica en la forma de afrontar los problemas, cuando se utiliza de una forma correcta, lo cual es de suma importancia en el proceso de educación y aun más en la asignatura de las Ciencias Naturales, las lecciones magistrales brindan al estudiante la oportunidad de ser motivado por quienes ya son expertos en el conocimiento. 
Matriz $\mathbf{N}^{\circ} 6$

Técnicas y recursos didácticos propuestos por los estudiantes

\begin{tabular}{|l|l|}
\hline \multicolumn{1}{|c|}{ Estudiante } & \multicolumn{1}{c|}{ Técnicas /recursos didácticos } \\
\hline $1,3,6,7,8,9,10,11,12,15,16$, & Juegos didácticos \\
$20,21,22$ & \\
\hline $1,2,3,4,9,11,15,16,19,24$ & Demostraciones experimentales \\
\hline $1,5,10,11,18,16,21,23,24,25$ & Experimentos \\
\hline $1,2,6,7,8,12,18,24$ & Videos \\
\hline $1,6,7,8,20,24$ & Presentaciones en PowerPoint \\
\hline $1,14,16,20,24$ & Exposiciones \\
\hline $1,18,16,20$, & Clases magistrales \\
\hline $1,9,10,11$, & Discusiones grupales \\
\hline $1,21,22$ & Filminas \\
\hline $1,14,20$ & Rotafolios \\
\hline 1 & Carteles \\
\hline
\end{tabular}

Fuente: Cuestionario para estudiantes.

Actualmente en la mayoría de los casos, el docente se centra en la trasmisión de los conocimientos, acompañado de otras técnicas que promueven muy poco la participación del estudiante en la construcción de su conocimiento, convirtiéndolo en un participante pasivo, teniendo como consecuencia la vinculación de los contenidos como una experiencia monótona y poco provechosa. Por ello, los estudiantes recomiendan otras técnicas y recursos didácticos más dinámicos e interactivos donde ellos sean partícipes y constructores de su propio conocimiento, es decir, donde su función sea más activa y se deje de lado las tradicionales clases magistrales (matriz $\mathrm{N}^{\circ} 6$ ), porque cuando estas nuevas técnicas y recursos son incluidas en la metodología, la lección resulta atractiva para el discente ya que provoca interés, participación, desafío intelectual y en algunos casos alto nivel de los procesos mentales, buscando de cierta forma el efecto sinérgico Zeigarnik-Hawthorne, es decir, producir motivación a través de una sensación de desafío por parte del profesor (Titone, 1981). 
Tabla $N^{\circ} 1$

Respuestas más significativas test de motivación para una grupo de noveno año de un colegio privado

\begin{tabular}{|l|c|c|c|c|c|}
\hline \multirow{2}{*}{ Pregunta } & \multicolumn{5}{|c|}{ Número de respuesta de los estudiantes } \\
\cline { 2 - 6 } & $\begin{array}{c}\text { Muy de } \\
\text { acuerdo }\end{array}$ & $\begin{array}{c}\text { De } \\
\text { acuerdo }\end{array}$ & $\begin{array}{c}\text { No estoy } \\
\text { seguro }\end{array}$ & $\begin{array}{c}\text { En } \\
\text { desacuerdo }\end{array}$ & $\begin{array}{c}\text { Muy en } \\
\text { desacuerdo }\end{array}$ \\
\hline $\begin{array}{l}\text { Me gustaría infor- } \\
\text { marme/aprender más } \\
\text { sobre esta unidad. }\end{array}$ & 2 & 12 & 7 & 1 & 1 \\
\hline $\begin{array}{l}\text { Me gustaría que hubi- } \\
\text { era más clases sobre } \\
\text { esta materia. }\end{array}$ & 2 & 9 & 7 & 2 & 3 \\
\hline $\begin{array}{l}\text { Me gustaría dedicar } \\
\text { más tiempo a esta ma- } \\
\text { teria en el escuela. }\end{array}$ & 2 & 9 & 9 & 2 & 1 \\
\hline $\begin{array}{l}\text { El trabajo me parece } \\
\text { interesante. }\end{array}$ & 6 & 10 & 6 & 0 & 1 \\
\hline $\begin{array}{l}\text { El trabajo no tiene } \\
\text { relación con mi vida. }\end{array}$ & 2 & 8 & 8 & 5 & 3 \\
\hline $\begin{array}{l}\text { Los materiales son } \\
\text { muy interesantes. }\end{array}$ & 3 & 12 & 5 & 2 & 1 \\
\hline
\end{tabular}

Fuente: Test de escala de actitudes para el estudiante

El test de motivación nos señala que dentro del grupo existe una motivación moderada hacia la materia de Ciencias ya que son pocas veces cuando los estudiantes se encuentran motivados debido a que la metodología utilizada por el profesor siempre es tradicional. Además parte de esta motivación es extrínseca porque el alumno concibe el aprendizaje como un instrumento para lograr una meta, la cual es una estabilidad económica futura, pero no existe una motivación intrínseca real hacia los contenidos que le permitan comprender y explicar el contexto que los rodea, puesto que las metodologías propiciadas por el profesor no siempre permiten un aprendizaje constructivista. (Matriz $\mathrm{N}^{\circ}$ 5) ni la vinculación del conocimiento con la realidad.

Cada una de las opiniones obtenidas a partir de los diferentes instrumentos de información aplicados concuerdan en que el nivel de motivación de los estudiantes en la mayoría de los casos depende de la variedad de técnicas, recursos y metodologías que utilice el docente debido a que de esta forma las clases no serán rutinarias. Cabe destacar que no existe una única metodología ni una técnica que tenga 
resultados favorables para todos los estudiantes, pero con una verdadera planificación, adecuación de las habilidades y del contexto tanto de los alumnos como del grupo en general, se logrará un aprendizaje significativo en ellos.

A través de los resultados obtenidos, se determinó que parte de la motivación de los estudiantes se encuentra influenciada por el tipo de metodología con que el docente desarrolla sus lecciones, por esto se pretendió desarrollar una metodología menos rígida y más dinámica e interactiva, con el fin de que los estudiantes obtengan un aprendizaje significativo.

Además, en esta propuesta se tomarán en cuenta las opiniones de los alumnos en la planificación de las actividades, recursos didácticos y técnicas didácticas porque ellos expresan más participación y dinamismo por parte del estudiante a través de experimentos, vídeos, demostraciones experimentales, giras educativas, entre otros. De esta manera, se dejará de lado la enseñanza memorística y conductista para buscar la comprensión, criticidad y la aplicación de los contenidos en la vida cotidiana.

A continuación se presentan técnicas y recursos didácticos que podrían ser implementados en una metodología constructivista y las habilidades que ayudan a que el estudiante desarrolle motivación para el aprendizaje. Además, se muestran ejemplos para el tema de la célula. 
La relación existente entre la metodología utilizada por un profesor de ciencias y la motivación que presentan

Tabla $\mathbf{N}^{\circ} 2$

Descripción de las técnicas y recursos didácticos recomendados para fomentar la motivación

\begin{tabular}{|c|c|c|c|}
\hline $\begin{array}{l}\text { Recurso/ } \\
\text { técnica }\end{array}$ & Descripción & $\begin{array}{l}\text { Habilidades a } \\
\text { desarrollar: }\end{array}$ & Actividad recomendada \\
\hline 兽 & $\begin{array}{l}\text { El juego es una forma de } \\
\text { explorar y conocer la realidad, } \\
\text { a través del gozo, el placer } \\
\text { y lo lúdico favoreciendo el } \\
\text { desarrollo del pensamiento, la } \\
\text { imaginación y la creatividad. }\end{array}$ & $\begin{array}{l}\text { Enseña a manejar el } \\
\text { ambiente que le rodea, } \\
\text { desarrollo de la personalidad } \\
\text { y de relaciones intra e } \\
\text { interpersonales }\end{array}$ & Bingo "La Célula" \\
\hline 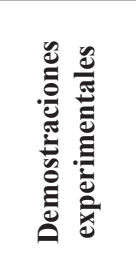 & $\begin{array}{l}\text { Por lo general, es llevada a } \\
\text { cabo por el docente, el cual } \\
\text { presenta una situación práctica } \\
\text { que da a conocer un resultado, } \\
\text { para ilustrar un principio o } \\
\text { fenómeno sin utilizar equipo } \\
\text { especializado. }\end{array}$ & $\begin{array}{l}\text { Comunicación entre profesor } \\
\text { y los estudiantes, ilustra un } \\
\text { concepto físico, motivación } \\
\text { en el estudio de un fenómeno } \\
\text { y amplía ideas cuando } \\
\text { el alumno participa en el } \\
\text { diseño. }\end{array}$ & Demostración de ósmosis \\
\hline 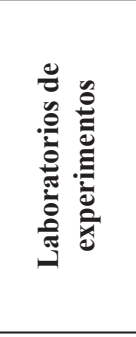 & $\begin{array}{l}\text { Son ensayos elaborados que } \\
\text { se realizan a través de un } \\
\text { procedimiento establecido, } \\
\text { que conlleva a una } \\
\text { observación sistemática en la } \\
\text { obtención de resultados }\end{array}$ & $\begin{array}{l}\text { Aprendizaje de conceptos y } \\
\text { principios científicos, destrezas } \\
\text { en la relación con aparatos } \\
\text { e instrumentos, actitudes } \\
\text { científicas, criticidad y } \\
\text { creatividad. Habilidades para } \\
\text { la investigación: observación, } \\
\text { formulación de hipótesis, } \\
\text { registro de datos, conclusiones. }\end{array}$ & $\begin{array}{l}\text { Experimento de osmosis: } \\
\text { "Quitando las arrugas a las } \\
\text { pasas" }\end{array}$ \\
\hline 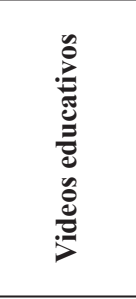 & $\begin{array}{l}\text { Un video es un sistema de } \\
\text { grabación y reproducción de } \\
\text { imágenes, que pueden estar } \\
\text { acompañadas de sonidos, } \\
\text { estos pueden tener utilidad } \\
\text { en educación, pueden ser: } \\
\text { documentales, lecciones, } \\
\text { narrativos, entre otros. }\end{array}$ & $\begin{array}{l}\text { Proporciona una visión } \\
\text { general del tema, facilita } \\
\text { el trabajo en equipo, } \\
\text { motivación y visualización } \\
\text { de conceptos abstractos }\end{array}$ & $\begin{array}{l}\text { La célula (2009), } \\
\text { Documental de la BBC }\end{array}$ \\
\hline 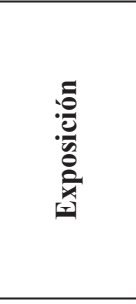 & $\begin{array}{l}\text { Consiste en la presentación } \\
\text { oral principalmente sobre } \\
\text { un tema, la cual debe estar } \\
\text { estructurada y debe presentar } \\
\text { los puntos más importantes } \\
\text { de la información }\end{array}$ & $\begin{array}{l}\text { Aprendizaje de conceptos, } \\
\text { participación del estudiantes } \\
\text { en el proceso educativo, } \\
\text { mejora la relación estudiante } \\
\text { profesor y estudiante-grupo, } \\
\text { desarrollo de habilidades } \\
\text { sociales, interpersonales y de } \\
\text { expresión }\end{array}$ & $\begin{array}{l}\text { Exposición grupal acerca de } \\
\text { los componentes de la célula } \\
\text { y las organelas }\end{array}$ \\
\hline 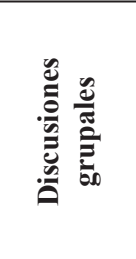 & $\begin{array}{l}\text { Es un dialogo en el cual se } \\
\text { expresan y comparten ideas, } \\
\text { opiniones y conocimientos } \\
\text { sobre un tema, para llegar } \\
\text { a una conclusión y la } \\
\text { resolución de un problema. }\end{array}$ & $\begin{array}{l}\text { Pensamiento crítico, } \\
\text { propiciar la creatividad y } \\
\text { participación, aprovecha } \\
\text { los conocimientos y } \\
\text { la experiencia de los } \\
\text { participantes y estimula la } \\
\text { reflexión y análisis. }\end{array}$ & $\begin{array}{l}\text { Comparación del } \\
\text { funcionamiento de una célula } \\
\text { con un fábrica industrial }\end{array}$ \\
\hline
\end{tabular}


Mediante la implementación de las actividades y recursos didácticos descritos anteriormente, se pretende crear metodologías que busquen un cambio, sin embargo, para que este cambio tenga efecto en el contexto educativo, se requiere que los profesores conozcan y dominen diversos abordajes metodológicos, no solo a nivel epistemológico sino que se convierta en una herramienta de uso frecuente al servicio de estudiantes y futuros ciudadanos. La importancia de cada una de las técnicas aplicadas hace más vivencial el aprendizaje del estudiante por lo que da una visión más amplia acerca de la ciencia en estudio.

Cabe destacar que la metodología utilizada por un profesor debe ser planificada de acuerdo con el contexto en que se desarrollan las clases, por ello esta propuesta es una recomendación, puesto que no existe una única metodología con resultados totalmente eficaces.

\section{Conclusiones}

De acuerdo con la investigación realizada se puede concluir que:

- $\quad$ Se determinó que la técnica didáctica más utilizada por el profesor de ciencias en el momento de impartir las lecciones es la expositiva "magistral".

- La motivación en el proceso de enseñanza-aprendizaje se logra con la utilización de métodos, procedimientos y estrategias que despierten el interés del alumno al aprender.

- Los estudiantes esperan que las lecciones de Ciencias sean más activas, que ofrezcan un mayor número de juegos didácticos, demostraciones y experimentos.

\section{Referencias bibliográficas}

Ávila, R., Cascante, P. \& Quesada, S. (2008). Diseño de módulos de capacitación para optimizar los procesos de enseñanza y aprendizaje para los docentes de ciencias y biología utilizando los recursos físicos y naturales disponibles en la estación biológica 28 millas para los colegios aledaños a la zona. Tesis de licenciatura no publicada, Universidad Nacional, Heredia, Costa Rica.

Díaz, F \& Hernández, G. (2002) Estrategias Docentes para un Aprendizaje Significativo. Una interpretación constructivista. México: McGraw Hill. 
La relación existente entre la metodología utilizada por un profesor de ciencias y la motivación que presentan los estudiantes de un grupo de noveno año

Diccionario de la Real Academia Española. (2001). Diccionario de la lengua española. Recuperado el 29 de marzo de 2011, de http://www.rae.es/rae.html.

Elliot, J. (2000). La investigación-acción en educación. España: Morata.

Esquivel, M., Gutiérrez, C., Solís, H. \& Zúñiga, G. (2005). La influencia que tiene el tipo de metodología empleada por el docente de Ciencias, Francés e Inglés sobre la motivación de los estudiantes del noveno años del Colegio María Inmaculada. Tesis de licenciatura no publicada, Universidad Nacional, Heredia, Costa Rica.

Fernández, L \& Orozco, A. (2002). La metodología empleada en la enseñanza de los estudios sociales y la relación con la motivación de los estudiantes de decimo año del colegio nocturno Miguel Obregón. Tesis de licenciatura no publicada, Universidad Nacional, Heredia, Costa Rica.

Gagné, R \& Briggs, L. (1997). La planificación de la enseñanza. Sus Principios. México: Trillas.

Gilbert, I, (2005).Motivar para aprender en el aula, las siete claves de la motivación escolar. España: Paidos.

Giroux, H. (1990). Los profesores como intelectuales: hacia una pedagogía crítica del aprendizaje. Argentina: Paidós.

Grisolía, M. (s.f.). ¿Qué es un recurso didáctico? Recuperado el 8 de abril de 2011, de http://webdelprofesor.ula.ve/humanidades/marygri/recursos.php

Hernández, R; Fernández, C; \& Baptista, P. (2006). Metodología de la Investigación. México: McGraw Hill.

Hidalgo, Y.\& Miranda, G. (2007). Influencia de las técnicas didácticas en la motivación que tengan los estudiantes hacia las clases de física de quinto año en dos colegios públicos costarricenses. Tesis de licenciatura no publicada, Universidad Nacional, Heredia, Costa Rica.

Martínez, (s.f.). La motivación. Recuperado el 10 de mayo de 2011, de http://www.uhu.es/cine.educacion/didactica/0083 motivacion. htm\#La_motivaci\%C3\%B3n_para_el_aprendizaje

Minnick, C. \& Alvermann C. (1994). Una didáctica de las ciencias. Procesos y aplicaciones. Argentina: AIQUE grupo editor S.A.

Ministerio de Educación Pública de Costa Rica. (2005).Programas de Estudio de Biología. San José: Autor.

Novak, J. D. (1982). El Papel Fundamental de la Teoría del Aprendizaje en una Teoría de la Instrucción. Madrid: Alianza.

Perkins, D. (2008). La escuela inteligente: del adiestramiento de la memoria a la educación de la mente. España: Gedisa.

Prot, B. (2005). Pedagogía de la motivación, cómo despertar el deseo de aprender. España: Narcea.

Titone, R. (1981). Metodología Didáctica. Madrid: Rialp. 